\title{
Editorial 3rd Intercontinental Cooperative ITP Study Group (ICIS) Expert Meeting in Basel, Switzerland, September 2009
}

\author{
Thomas Kühne • Paul Imbach
}

Received: 22 March 2010 /Accepted: 23 April 2010 /Published online: 7 May 2010

(C) Springer-Verlag 2010

On September 3-5, 2009, the 3rd Intercontinental Cooperative ITP Study Group (ICIS) Expert Meeting was held in Basel, Switzerland. After Bürgenstock (2003) and Yverdon (2006), the meeting is gaining a status of tradition. More than 100 experts involved in basic science and clinical research met to discuss consensus issues and development of strategies in immune thrombocytopenia (ITP). This issue of Annals of Hematology represents a compilation of lectures and working group discussions. The four working groups "Basic science", "Management endpoints of ITP", "Chronic ITP", and "Future research: resources and regulations" were preceded by lectures of the same topic. Speakers were invited to send a review article of their lecture. The articles of Clemetson and Panzer, Tamary et al., Grainger et al., and Zehnder et al. represent summaries of the working group discussions, which were submitted after the meeting and after consensus work of co-authors and participants.

The ICIS Expert Meeting was a great opportunity to exchange new knowledge and to plan for the future. "Basic science" is reflected by the articles of Biburger et al., Pang and Lazarus, Semple, and Speer and Schmugge. Neunert, and Klaassen and Young wrote an article evaluating bleeding for "Management endpoints", and Godeau and Michel, BoltonMaggs and Kok, Kühne and Imbach, Jawa et al. and Wadenvik and Olsson wrote for the group "Chronic ITP". Bergmann et al. wrote an article about genetics in pediatric ITP for the group "Future research".

Immune thrombocytopenia, formerly called idiopathic or immune thrombocytopenic purpura is a bleeding diathesis

T. Kühne $(\bowtie) \cdot$ P. Imbach

Division of Oncology/Hematology,

University Children's Hospital,

Postfach, Römergasse 8,

CH-4005 Basel, Switzerland

e-mail: Thomas.Kuehne@ukbb.ch with the hallmark of thrombocytopenia, resulting from premature platelet destruction by the monocytic-phagocytic system and by certain degree of platelet production failure. In a majority of patients, autoantibodies of various specificities can be detected, however, not in every patient, which indicates heterogeneity of the etiology and immune pathophysiology in patients with ITP. It is this heterogeneity which may be responsible for different degrees of responses to therapeutic interventions, and also for the many debates and controversies around ITP. The terminology, definitions, and the practice guidelines characterizing ITP have recently been improved by the international harmonization report [1] and by the international consensus report [2]. However, there are many open questions which have been addressed by ICIS since more than 12 years. ICIS was founded by several hematologists to establish a network of scientists and physicians involved in the field of ITP. Several registries have been developed, of which two are closed and published (Registry I 1997-2000 with 2,786 patients and Registry II 2002-2004 with 1,388 patients). Please visit www.itpbasel.ch for references. Currently, there are two registries open: the Pediatric and Adult Registry on Chronic ITP since 2004 with 2,040 patients and the Splenectomy Registry (since 1998 with 174 patients). Within the 12-year experience of ICIS registries, we learned how to formulate registry questions, electronic data management including online processes, and data quality assessments. Data control and evaluation need cautiousness and high professional experience. Scientific results and publications have to be peer-reviewed by experts of registries. Ongoing communication and discussion through regular international meetings support this process.

Attending experts, speakers, and manuscript authors did a great job and made our meeting a fruitful and successful event. Our thanks go to all active participants of the 
meeting and to all ICIS investigators. Warm thanks go to Verena Stahel, Caroline Martin, and Monika Imbach, our ICIS office staff, as well as to Erika Scheibli, secretary, who are all wonderful organizers. The research activities of ICIS are supported by the ITP Foundation founded by family McGuire, the Eduard Waeffler-Ludwig Stiftung Basel Fund, and the University Children's Hospital Basel, as well as industrial support by Amgen, Biotest, CSL Behring, F. Hoffmann-La Roche, GlaxoSmithKline, and PPTA.

ITP reflects since its description by Paul Gottlieb Werlhof a fast-changing field. It becomes clearer that it is not a disorder, not Werlhof's disease, but rather an immunological phenomenon caused by different factors and mechanisms. We hope that such ICIS meetings stimulate the participants and all participating and interested investigators to maintain their enthusiasm and energy to further develop basic and clinical research which ultimately will help children and adults with ITP. All meeting participants and ICIS investigators can be seen by visiting our homepage www.itpbasel.ch.

\section{References}

1. Rodeghiero F et al (2009) Standardization of terminology, definitions and outcome criteria in immune thrombocytopenic purpura (ITP) of adults and children. Report from an international working group. Blood 113:2386-2393

2. Provan et al (2010) International consensus report on the investigation and management of primary immune thrombocytopenia. Blood $115: 168-186$ 